\title{
Nonlinear Microwave Imaging for Breast-Cancer Screening Using Gauss-Newton's Method and the CGLS Inversion Algorithm
}

Rubæk, Tonny; Meaney, P. M.; Meincke, Peter; Paulsen, K. D.

Published in:

I E E E Transactions on Antennas and Propagation

Link to article, DOI:

10.1109/TAP.2007.901993

Publication date:

2007

Document Version

Publisher's PDF, also known as Version of record

Link back to DTU Orbit

Citation (APA):

Rubæk, T., Meaney, P. M., Meincke, P., \& Paulsen, K. D. (2007). Nonlinear Microwave Imaging for BreastCancer Screening Using Gauss-Newton's Method and the CGLS Inversion Algorithm. I E E E Transactions on Antennas and Propagation, 55(8), 2320 - 2331. https://doi.org/10.1109/TAP.2007.901993

\section{General rights}

Copyright and moral rights for the publications made accessible in the public portal are retained by the authors and/or other copyright owners and it is a condition of accessing publications that users recognise and abide by the legal requirements associated with these rights.

- Users may download and print one copy of any publication from the public portal for the purpose of private study or research.

- You may not further distribute the material or use it for any profit-making activity or commercial gain

- You may freely distribute the URL identifying the publication in the public portal 


\title{
Nonlinear Microwave Imaging for Breast-Cancer Screening Using Gauss-Newton's Method and the CGLS Inversion Algorithm
}

\author{
Tonny Rubæk, Student Member, IEEE, Paul M. Meaney, Member, IEEE, Peter Meincke, Member, IEEE, and
} Keith D. Paulsen, Member, IEEE

\begin{abstract}
Breast-cancer screening using microwave imaging is emerging as a new promising technique as a supplement to X-ray mammography. To create tomographic images from microwave measurements, it is necessary to solve a nonlinear inversion problem, for which an algorithm based on the iterative Gauss-Newton method has been developed at Dartmouth College. This algorithm determines the update values at each iteration by solving the set of normal equations of the problem using the Tikhonov algorithm. In this paper, a new algorithm for determining the iteration update values in the Gauss-Newton algorithm is presented which is based on the conjugate gradient least squares (CGLS) algorithm. The iterative CGLS algorithm is capable of solving the update problem by operating on just the Jacobian and the regularizing effects of the algorithm can easily be controlled by adjusting the number of iterations. The new algorithm is compared to the Gauss-Newton algorithm with Tikhonov regularization and is shown to reconstruct images of similar quality using fewer iterations.
\end{abstract}

Index Terms-Biomedical electromagnetic imaging, cancer, electromagnetic scattering inverse problems, image reconstruction, imaging, inverse problems, microwave imaging, nonlinear equations.

\section{INTRODUCTION}

$\mathbf{M}$ ICROWAVE imaging is emerging as a promising new technique for use in breast-cancer screening [1]-[5]. The use of microwave imaging as a supplement or alternative to the widely used X-ray mammography is considered to be appealing because of the nonionizing nature of the microwaves and because the physical parameters providing contrast in the microwave images are different from those in the X-ray images. This implies that microwave imaging may be useful for detecting tumors that are not visible in X-ray mammography.

The techniques currently applied for microwave imaging of the breast can be divided into two categories. In the first, radarbased approaches are used. This involves transmitting a broadband pulse into the breast and creating images by use of time-re-

Manuscript received October 12, 2006; revised January 26, 2007. The work of T. Rubæk and P. Meincke was supported by the Danish Technical Research Council and the work of P. M. Meaney and K. D. Paulsen was supported by the NIH/NCI under Grant P01-CA80139.

T. Rubæk and P. Meincke are with the Technical University of Denmark, DK-2800 Kgs. Lyngby, Denmark (e-mail: tru@ oersted.dtu.dk).

P. M. Meaney and K. D. Paulsen are with Thayer School of Engineering, Dartmouth College, Hanover, NH 03755 USA.

Digital Object Identifier 10.1109/TAP.2007.901993 versal algorithms, thereby synthetically focusing the transmitted pulse at different locations within the breast [1], [6], [7].

The other major category is based on tomographic imaging using nonlinear inversion in which a forward model is created using Maxwell's equations [8], [9]. When using the tomographic techniques, the breast is irradiated by one antenna and the response is measured by a number of receiving antennas. By alternating which antenna is transmitting the signal, illumination from all directions can be achieved. These measurements are then inserted into a forward model based on the frequency-domain form of Maxwell's equations which, in turn, can be inverted to obtain the constitutive-parameter distribution of any target inside the imaging domain. The forward model based on Maxwell's equations leads to a nonlinear ill-posed inversion problem and implies that advanced signal processing techniques are necessary to obtain an image from the measurement of the transmit-receive data.

At Dartmouth College, a Gauss-Newton iterative method using a Tikhonov regularization for solving for the updates (GN-T) is applied for solving the nonlinear imaging problem. This method involves solving a forward problem at each iteration and constructing a Jacobian matrix $\underline{J}$ from the forward problem to update the values of the constitutive parameters in the imaging domain. The update values are found by solving the normal equation to an under-determined linear problem at each iteration of the GN-T algorithm. This procedure requires the explicit calculation of the matrix $\underline{J}^{T} \underline{J}$. Because of the ill-posedness of the underlying problem, regularization is needed for which a Tikhonov algorithm [10, Sec. 5.1] is applied. The speed of the imaging algorithm is governed primarily by two factors, one being the speed of the forward solver, and the other being the ability of the algorithm to update the values of the constitutive parameters as accurately as possible, allowing for fewer calls to be made to the forward solver [11], [12].

The new algorithm for updating the values of the constitutive parameters described in this paper addresses the latter of these factors. The new algorithm uses the conjugate gradient least squares (CGLS) algorithm [10, Sec. 6.3] for calculating the updates at each iteration of the Gauss-Newton algorithm. The CGLS algorithm is an iterative algorithm for solving linear equations and determines the solution of the linear problem by projecting it into a Krylov subspace. The algorithm does not need the explicit calculation of the matrix $\underline{J}^{T} \underline{J}$ but is capable of working directly on the Jacobian matrix. Furthermore, the regularizing effects of the CGLS algorithm are governed by the 


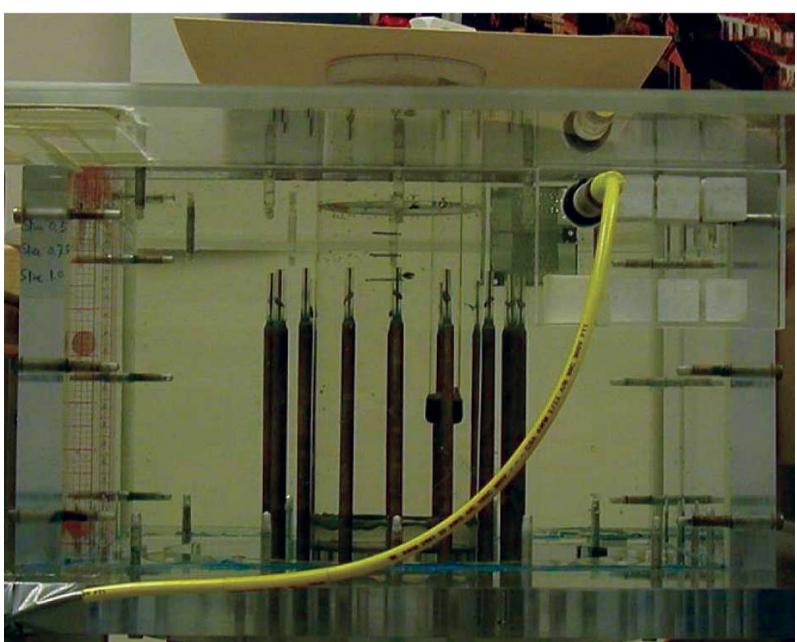

Fig. 1. Photo of imaging system. The monopoles are positioned in a circular setup and during the measurements, the tank is filled with a coupling liquid.

number of iterations the algorithm is allowed to run, thereby allowing for easier control over the regularizing effects than in the current GN-T algorithm.

This paper contains a brief description of the current imaging system in Section II, and an introduction to solving imaging problems using the Gauss-Newton method in Section III. This section also contains a description of the algorithm currently applied. In Section IV, the new algorithm is introduced based on the CGLS algorithm. Finally, in Section V, the new algorithm is tested and its performance is compared to that of the currently applied algorithm using a simulation, phantom measurements, and patient data.

\section{IMAGING SYSTEM}

The imaging system at Dartmouth College consists of 16 monopole antennas positioned in a circular array, as shown in Fig. 1, and is designed to operate over the frequency range from 500 to $2300 \mathrm{MHz}$. The patient lies prone on top of the measurement tank with the breast to be examined suspended through an aperture in the top of the tank as seen in the schematic in Fig. 2. The tank is filled with a coupling liquid, closely mimicking the average constitutive parameters of the breast [13], maximizing the amount of microwave energy coupled into the breast. A more thorough description of the imaging system is found in [14].

During the acquisition of data, the antenna array scans through seven vertical positions at $1 \mathrm{~cm}$ increments. At each plane, the antennas sequentially act as transmitters while the response is measured at the remaining 15 antennas. This results in 240 coherent measurements of the scattered field for each plane. Currently, the system operates by creating two-dimensional (2-D) slice images of each of the seven planes and the image reconstruction is based on the assumption that the scattering problem can be reasonably represented as a 2-D problem [11]. The validity of this assumption has been investigated in [15], wherein it was found that although the simplification of the imaging problem to 2-D does introduce some inaccuracies

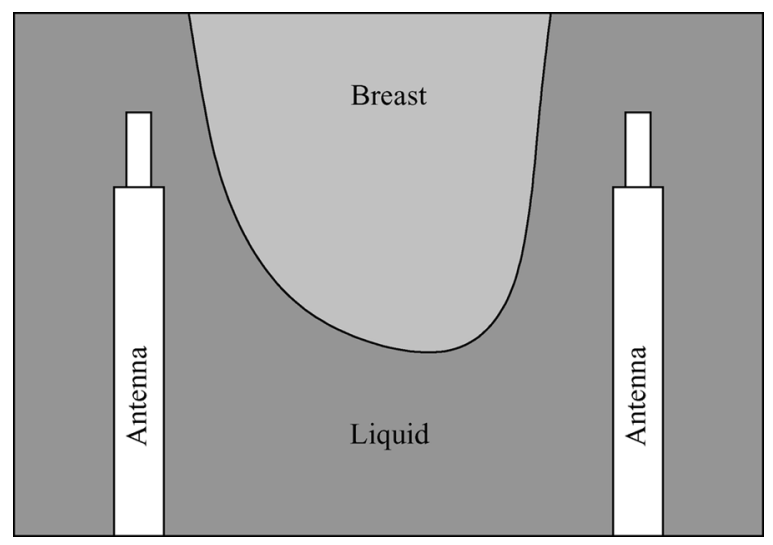

Fig. 2. Measurement setup. The antennas are moved downwards and measurements are taken at 7 planes through the breast, with plane 1 being closest to the chest and plane seven being closest to the nipple.

in the reconstructed images, the relatively small radius of the imaging system ensures that the inaccuracies are not critical.

\section{GAUSS-Newton's Method}

When reconstructing the microwave tomographic images, the distribution of the constitutive parameters in the imaging domain is represented by the complex wave number squared

$$
k^{2}(\boldsymbol{r})=\omega^{2} \mu_{0} \epsilon(\boldsymbol{r})+i \omega \mu_{0} \sigma(\boldsymbol{r})
$$

where the time notation $\mathrm{e}^{-i \omega t}$ is assumed. In this expression, $\epsilon$ is the permittivity, $\sigma$ is the conductivity, $\mu_{0}$ is the free-space permeability, and $\omega$ and $i$ are the angular frequency and complex unit, respectively. The vector $\boldsymbol{r}$ is a position vector in the imaging domain. The distribution of the squared wave numbers is determined by solving the minimization problem

$$
\underline{k}^{2}=\arg \min \left\{\left\|\underline{E}^{\text {meas }}-\underline{E}^{\text {calc }}\left(\underline{k}^{2}\right)\right\|_{2}^{2}\right\}
$$

where $\underline{E}^{\text {calc }}\left(\underline{k}^{2}\right)$ is a vector of field values calculated using the forward model for a given distribution of constitutive parameters stored in the vector $\underline{k}^{2}$, and $\underline{E}^{\text {meas }}$ is a vector of the corresponding measurements. The two-norm $\|\cdot\|_{2}$ of a $V$-element vector $\underline{x}$ is the square root of the sum of the squares of the elements in the vector given by

$$
\|\underline{x}\|_{2}=\sqrt{\sum_{j=1}^{V}\left|x_{j}\right|^{2}}
$$

where $|\cdot|$ indicates the complex modulus of the elements.

For this implementation, the image reconstruction is performed in terms of the relative change in phase and amplitude, the so-called log-phase $(1-p)$ representation [16], as opposed to changes in the absolute complex field values. The measured data is represented using the difference in the logarithm of the amplitude and the unwrapped phase between a measurement 
with an empty system and a measurement with a target, i.e., a breast, present. The measurement data is stored in a vector

$$
\underline{E}_{1-\mathrm{p}}^{\text {meas }}=\left[\begin{array}{c}
\log \left|\underline{E}_{\text {target }}^{\text {meas }}\right|-\log \left|\underline{E}_{\text {empty }}^{\text {meas }}\right| \\
\text { phase } \underline{E}_{\text {target }}^{\text {meas }}-\text { phase } \underline{E}_{\text {empty }}^{\text {meas }}
\end{array}\right]
$$

that is twice the length of the original complex measurement vector, $\underline{E}^{\text {meas }}$. The calculated data is reconfigured in a similar way, yielding the vector

$$
\underline{E}_{1-\mathrm{p}}^{\mathrm{calc}}\left(\underline{k}^{2}\right)=\left[\begin{array}{c}
\log \left|\underline{E}^{\text {calc }}\left(\underline{k}^{2}\right)\right|-\log \left|\underline{E}^{\text {calc }}\left(\underline{k}_{0}^{2}\right)\right| \\
\operatorname{phase} \underline{E}^{\text {calc }}\left(\underline{k}^{2}\right)-\operatorname{phase} \underline{E}^{\text {calc }}\left(\underline{k}_{0}^{2}\right)
\end{array}\right] .
$$

In this expression, $\underline{k}_{0}^{2}$ denotes the known distribution of constitutive parameters when there is no target present in the system, i.e., the constitutive parameters of the coupling liquid which can easily be measured using a commercially available probe kit. At Dartmouth College, an Agilent Network Analyzer (E5071C) with an Agilent Dielectric Probe Kit (85070E) is used.

The minimization problem to be solved for reconstructing the images can now be rewritten using the new vectors as

$$
\underline{k}^{2}=\arg \min \left\{\left\|\underline{E}_{\mathrm{l}-\mathrm{p}}^{\text {meas }}-\underline{E}_{\mathrm{l}-\mathrm{p}}^{\mathrm{calc}}\left(\underline{k}^{2}\right)\right\|_{2}^{2}\right\} .
$$

The use of the logarithm of the magnitude and unwrapped phase has been shown to emphasize the large relative changes observed at the antennas on the opposite side of the breast, i.e., within the main projection of the target, effectively containing more pertinent information about the scattering problem. At the same time, the signals with higher absolute magnitude measured by the antennas close to the transmitter, in which the object-induced changes are relatively small, are given less weight [16].

The calculation of the forward solution $\underline{E}_{1-p}^{c a l c}$ is based on the 2-D form of Maxwell's equations, yielding a nonlinear optimization problem for which the Gauss-Newton method is applied [17, Ch. 4-6], [18]. Hence, it is assumed that the nonlinear expression for the field as a function of the distribution of squared wave numbers can be approximated locally by a first-order Taylor expansion as

$$
\underline{E}_{1-\mathrm{p}}^{\mathrm{calc}}\left(\underline{k}_{n+1}^{2}\right)=\underline{E}_{\mathrm{l}-\mathrm{p}}^{\mathrm{calc}}\left(\underline{k}_{n}^{2}\right)+\underline{\underline{J}}\left(\underline{k}_{n}^{2}\right) \Delta k_{n}^{2}
$$

where $\underline{\underline{J}}$ is the Jacobian matrix and

$$
\underline{\Delta k_{n}^{2}}=\underline{k}_{n+1}^{2}-\underline{k}_{n}^{2}
$$

with $n$ being the current iteration number. In this form, the minimization problem can be reformulated as a number of local linear minimization problems given by

$\underline{k}_{n+1}^{2}=\arg \min \left\{\left\|\underline{E}_{1-\mathrm{p}}^{\text {meas }}-\left(\underline{E}_{1-\mathrm{p}}^{\mathrm{calc}}\left(\underline{k}_{n}^{2}\right)+\underline{\underline{J}}\left(\underline{k}_{n}^{2}\right) \underline{\Delta k_{n}^{2}}\right)\right\|_{2}^{2}\right\}$.
The iterative Gauss-Newton method currently applied at Dartmouth College consists of five steps in each iteration.

1) The forward model is used to calculate the electric fields from the distribution of constitutive parameters $\underline{k}_{n}^{2}$ and to check for termination of the algorithm.

2) Calculate the Jacobian $\underline{\underline{J}}$ for the current property distribution, $\underline{k}_{n}^{2}$.

3) Obtain the Newton direction $\left[18\right.$, Sec. 1.6] $\underline{d}_{n}$ by solving the linear problem

$$
\underline{\underline{J}}\left(\underline{k}_{n}^{2}\right) \underline{d}_{n}=\underline{E}_{1-\mathrm{p}}^{\text {meas }}-\underline{E}_{1-\mathrm{p}}^{\mathrm{calc}}\left(\underline{k}_{n}^{2}\right)
$$

using the normal equation and the Tikhonov regularization algorithm.

4) Determine the Newton step $\left[18\right.$, Sec. 1.6] $\alpha_{n}$ satisfying

$$
\alpha_{n}=\arg \min \left\{\left\|\underline{E}_{1-\mathrm{p}}^{\text {meas }}-\underline{E}_{1-\mathrm{p}}^{\mathrm{calc}}\left(\underline{k}_{n}^{2}+\alpha_{n} \underline{d}_{n}\right)\right\|_{2}^{2}\right\} .
$$

5) Update the values of the constitutive parameters using

$$
\underline{\Delta k_{n+1}^{2}}=\underline{k}_{n}^{2}+\alpha_{n} \underline{d}_{n}
$$

The operations listed above can be divided into two categories. Steps 1 and 2 concern the forward calculations while steps 3 through 5 concern the computation of the new values. The most time consuming part of the iterations of the GN-T algorithm is the forward calculations in steps 1 and 2 which take approximately 1 min per iteration. The computation of the update, on the other hand, takes approximately $1 \mathrm{~s}$. The new CGLS-based algorithm, to be described in Section IV, focuses mainly on improving steps 3 through 5, and aims to reduce the overall time consumption by reducing the number of iterations, and thereby calls to the forward solver, needed for the algorithm to converge. A review of the current implementation of the Gauss-Newton algorithm is presented in the following section.

\section{A. Forward Solver and Jacobian}

The forward solver used in the algorithm is a hybrid-element algorithm which uses the finite-element method for representing the electromagnetic scattering problem within the heterogeneous imaging domain and a boundary-element method for representing the homogeneous area outside of the imaging domain [19]. The values of the constitutive parameters are reconstructed on a coarse mesh, in this case with 559 nodes as shown in Fig. 3(a), which are subsequently interpolated onto a finer finite-element mesh, in this case with 3903 nodes as shown in Fig. 3(b), for computation by the forward solver [20].

The influence of the antennas not acting as transmitter or receiver being present in the imaging system is accounted for by representing them as electromagnetic sinks within the surrounding boundary-element zone as described in [21], [22]. The hybrid-element approach is useful as the forward solver here in that it is quite accurate because it does not require approximate boundary conditions. Its efficiency arises from the use of bounded matrix techniques facilitated by the finite-element approach and by the fact that only the target zone requires finite-element discretization [19]. In addition, incorporation of the adjoint technique [14] effectively reduces the calculation of the 


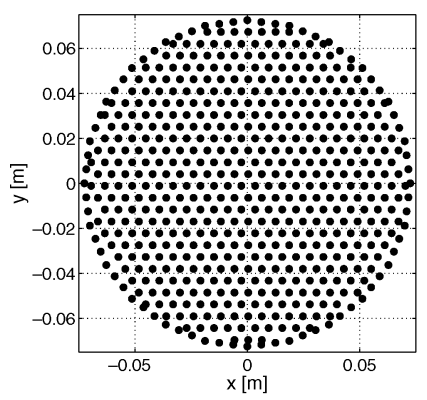

(a)

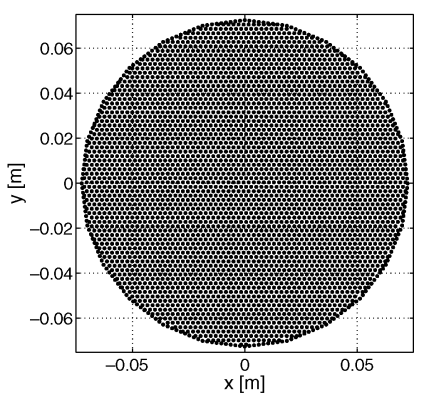

(b)
Fig. 3. Nodes of the coarse and fine meshes. The parameters are reconstructed on the coarse mesh and interpolated onto the finer mesh for computation by the forward solver. (a) Coarse mesh; (b) fine mesh.

Jacobian matrix to a set of simple inner product operations, reducing the overall computation time of this task to a fraction of that of the forward solution.

\section{B. Newton Direction}

The Newton direction $\underline{d}_{n}$ is currently found by solving the normal equation of the under-determined matrix equation (10). The normal equation is given in terms of the matrix $\underline{\underline{J}}^{T} \underline{\underline{J}}$ as

$$
\underline{\underline{J}}^{T} \underline{\underline{J}}^{\underline{d}} \underline{ }_{n}=\underline{\underline{J}}^{T}\left(\underline{E}_{1-\mathrm{p}}^{\mathrm{meas}}-\underline{E}_{1-\mathrm{p}}^{\mathrm{calc}}\left(\underline{k}_{n}^{2}\right)\right)
$$

where the argument of the Jacobian matrix has been omitted for improved readability. Since this problem is ill-conditioned, the Tikhonov algorithm [10, Sec. 5.1] is applied, yielding the linear equation

$$
\left(\underline{\underline{J}}^{T} \underline{\underline{J}}+\lambda \underline{\underline{I}}\right) \underline{d}_{n}=\underline{\underline{J}}^{T}\left(\underline{E}_{1-\mathrm{p}}^{\text {meas }}-\underline{E}_{1-\mathrm{p}}^{\text {calc }}\left(\underline{k}_{n}^{2}\right)\right)
$$

where the regularization parameter $\lambda$ is found using the method described in [23, Eq. (16)], wherein the trace of the matrix $\underline{\underline{J}}^{T} \underline{\underline{J}}$ is used as the basis of the calculation.

\section{Newton Step}

Although advanced algorithms exist for calculation of the Newton step size [17, Ch. 8], the fact that these algorithms all requires multiple calculations of the forward model to determine the optimum value of $\alpha_{n}$ means that they are not well suited for use in this algorithm where calculation of the forward model is the most time consuming operation. Instead, a simple yet effective method has been implemented in which the Newton step is set to a value determined by the iteration number. Using this method, the value of $\alpha_{n}$ is set to 0.1 during the first three iterations and then increased gradually in each of the following iterations until it reaches a value of 0.5 after 12 iterations. The small iteration step size has the primary benefit of ensuring relatively slow changes in the phase distribution of the computed fields between iterations thus acting to reduce the possibility of inducing complex nulls in the imaging domain. The avoidance of these nulls is crucial to the stability of this approach [24].

\section{Update of Values}

The values of the constitutive parameters are updated using the standard formulation

$$
\underline{k}_{n+1}^{2}=\underline{k}_{n}^{2}+\alpha_{n} \underline{d}_{n} .
$$

Depending on the noise level and degree of model mismatch, the updated values may contain high-frequency spatial variation. This is minimized by the application of a spatial-filtering algorithm that smooths the values of $\underline{k}_{n+1}^{2}$ by averaging them with a weighted sum of the values of the neighboring nodes [25, Appendix A].

\section{E. Termination of Algorithm}

In general, the algorithm converges within 13 to 15 iterations. In practice, especially while mass processing data from numerous patient exams, the algorithm is allowed to run 20 iterations to ensure convergence is reached.

\section{GAuSs-Newton CGLS ALgORITHM}

The new algorithm is focused on steps 3 to 5 in the Gauss-Newton algorithm, that is, determining the update at each iteration. To obtain a more efficient algorithm, the three steps have been merged into a single step based on the use of the CGLS algorithm [10, Sec. 6.3]. The new algorithm is denoted as the Gauss-Newton CGLS (GN-C) algorithm.

\section{A. CGLS Algorithm}

The CGLS algorithm is an iterative algorithm of the conjugate gradient type, and is applied for determining the update values at iteration $n$ in the GN-C algorithm $\underline{\Delta k_{n}^{2}}$ by solving the linear problem

$$
\begin{aligned}
& \underline{\Delta k}_{n}^{2}=\arg \min \left\{\| \underline{\underline{J}}\left(\underline{\Delta k}_{n}^{2}\right) \underline{\Delta k_{n}^{2}}\right. \\
& \left.-\left(\underline{E}_{1-\mathrm{p}}^{\text {meas }}-\underline{E}_{1-\mathrm{p}}^{\mathrm{calc}}\left({\underline{\Delta k_{n}^{2}}}_{n}^{2}\right)\right) \|_{2}^{2}\right\}
\end{aligned}
$$

and thus does not work on the normal equation. The solution to this linear equation after $m$ CGLS iterations ( $m$ CGLS iterations per each Gauss-Newton iteration $n$ ) is given by

$$
\left[\underline{\Delta}_{n}^{2}\right]^{(m)}=\arg \min \left\{\left\|\underline{\underline{J}}\left[\underline{\Delta}_{n}^{2}\right]^{(m)}-\left(\underline{E}_{1-\mathrm{p}}^{\text {meas }}-\underline{E}_{1-\mathrm{p}}^{\text {calc }}\right)\right\|_{2}^{2}\right\}
$$

subject to

$$
\left[\underline{\Delta}_{n}^{2}\right]^{(m)} \in \mathfrak{K}_{m}\left\{\underline{\underline{J}}^{T} \underline{\underline{J}},\left[\underline{\underline{J}}^{T}\left[\underline{E}_{1-\mathrm{p}}^{\text {meas }}-\underline{E}_{1-\mathrm{p}}^{\text {calc }}\right]\right]\right\}
$$

where $\mathfrak{K}_{m}$ is the $m$-dimensional Krylov subspace defined by the Jacobian matrix and the vector of the difference between the measured and calculated fields, and the arguments of the Jacobian and the calculated solution have been omitted for improved readability. The solution after $m$ iterations is thus the least-squares solution to the original problem projected into the $m$-dimensional Krylov subspace $\mathfrak{K}_{m}$. 
Each iteration of the CGLS algorithm is comprised of five simple steps [10, Eq. (6.14)], allowing for an efficient implementation of the algorithm. The algorithm is initialized with all elements of the update vector set to zero

$$
\left[\underline{\Delta k_{n}^{2}}\right]^{(0)}=\underline{0} .
$$

In addition to the update vector, the algorithm requires the residual vector $\underline{r}$ which is initialized as

$$
\underline{r}^{(0)}=\left(\underline{E}_{1-\mathrm{p}}^{\text {meas }}-\underline{E}_{1-\mathrm{p}}^{\text {calc }}\right)-\underline{\underline{J}}\left[\underline{\Delta k}_{n}^{2}\right]^{(0)}=\left(\underline{E}_{1-\mathrm{p}}^{\text {meas }}-\underline{E}_{1-\mathrm{p}}^{\text {calc }}\right)
$$

and an auxiliary vector $\underline{a}$ initialized by

$$
\underline{a}^{(0)}=\underline{\underline{J}}^{T} \underline{r}^{(0)} .
$$

At each iteration $m=1,2, \ldots$ of the CGLS algorithm, the value of the update vector $\left[\underline{\Delta k}_{n}^{2}\right]^{(m)}$ is computed using

$$
\left[\underline{\Delta}_{n}^{2}\right]^{(m)}=\left[\underline{\Delta k}_{n}^{2}\right]^{(m-1)}+\alpha_{m} \underline{a}^{(m-1)}
$$

and

$$
\alpha_{m}=\frac{\left\|\underline{\underline{J}}^{T} \underline{r}^{(m-1)}\right\|_{2}^{2}}{\left\|\underline{\underline{J}} \underline{\underline{a}}^{(m-1)}\right\|_{2}^{2}} .
$$

Thus, the solution is found as a linear combination of the vectors $\underline{a}^{(m)}$ where the weight of the individual vectors are found as the ratio between the squared two-norms of the matrix products of the Jacobians and the residual and auxiliary vectors, respectively. The residual and auxiliary vectors are updated at each iteration using

$$
\begin{aligned}
& \underline{r}^{(m)}=\underline{r}^{(m-1)}-\alpha_{m} \underline{\underline{J}}^{(m-1)} \underline{a}^{(m-1)} \\
& \underline{a}^{(m)}=\underline{\underline{J}}^{T} \underline{r}^{(m)}+\beta_{m} \underline{\underline{a}}^{(m-1)}
\end{aligned}
$$

and

$$
\beta_{m}=\frac{\left\|\underline{\underline{J}}^{T} \underline{\underline{r}}^{(m)}\right\|_{2}^{2}}{\left\|\underline{\underline{J}}^{T} \underline{\underline{r}}^{(m-1)}\right\|_{2}^{2}} .
$$

It can be shown that the vectors obtained by the matrix products of the transposed Jacobian and the individual residual vectors $\underline{J}^{T} \underline{r}^{(m)}, m=1,2, \ldots$ are orthogonal to each other [10, Sec. 6.3]. This implies that the auxiliary vector $\underline{a}^{(m)}$ is updated by adding an orthogonal vector scaled by the ratio between the squared norms of the current and previous residuals $\beta_{n}$ to the previously used vector, which corresponds to adding a new dimension to the solution. This illustrates how each iteration of the CGLS algorithm adds a dimension to the Krylov subspace onto which the solution is projected as stated in (17b). It should be noted that in finite precision, the orthogonality of the residual vectors is progressively diminished as the number of iterations with the CGLS algorithm increases due to rounding errors. This implies that there is an effective upper limit on the number of dimensions which can be obtained for the Krylov subspace.
As described in [10, Sec. 6.3.2 and 6.4], the exact details of the regularizing effects of the CGLS algorithm are still not completely understood. It is, however, known that the solutions provided by the CGLS algorithm closely follow the L-curve [10, Sec. 4.6] of the more widely-used Tikhonov algorithm [10, Sec. 5.1], the L-curve being the norm of the solution $\left[\underline{\Delta k_{n}^{2}}\right]^{(m)}$ as a function of the norm of the residual $\underline{r}^{(m)}$. The first iterations in the CGLS algorithm correspond to a high value of the regularization parameter in the Tikhonov algorithm, whereas the results obtained as the number of iterations increases correspond to decreasing the value of the regularization parameter. In this way, the regularizing effects of the CGLS algorithm is governed by the number of iterations $m$ rather than by an explicit regularization parameter.

\section{B. Determining the Update Values}

Usually, when solving a linear problem, the desired solution is that for which the L-curve has the maximum curvature, also known as the corner of the L-curve [10, Sec. 7.5]. However, it has been found in this work that this is not necessarily the case when solving for updates in the nonlinear GN-C algorithm. Instead, the result obtained after only a few iterations of the CGLS algorithm, corresponding to an over-regularized solution, has been found to yield the best results. Further discussion on this topic is found in Section V-A.

A two-step procedure for determining the number of iterations of the CGLS algorithm, similar to that previously suggested in [25], has been developed based on the normalized two-norm $\eta_{n}$ given by

$$
\eta_{n}=\frac{\left\|\underline{E}_{1-\mathrm{p}}^{\text {meas }}-\underline{E}_{1-\mathrm{p}}^{\text {calc }}\left(\underline{k}_{n}^{2}\right)\right\|_{2}}{\left\|\underline{E}_{1-\mathrm{p}}^{\text {meas }}\right\|_{2}} .
$$

Early in the reconstruction process, only two iterations of the CGLS algorithm are needed to determine the update values. When the relative change in $\eta_{n}$, defined as

$$
\Delta \eta_{n}=\frac{\eta_{n+1}}{\eta_{n}}-1
$$

between two iterations is greater than $-10 \%$, the number of iterations in the CGLS algorithm is increased to 16 . This is based on the assumption that as the solution gets closer to the actual distribution of the constitutive parameters, the local linearization obtained by the first-order Taylor expansion is a better approximation to the actual problem than when the solution is far from the actual distribution. It should be noted that the value of $\Delta \eta_{n}$ is negative as long as the value of $\eta_{n}$ decreases, i.e., as long as the calculated data approach the measured data.

The termination of the Gauss-Newton algorithm is also based on the normalized two-norm $\eta$. The algorithm is terminated when $\Delta \eta_{n}$ obtains a value greater than $-3 \%$ or when the value of $\eta_{n}$ drops below $\eta_{\text {term }}=0.03$. These thresholds have been determined by trial and error and are dependent on the noise level in the system. In systems with more noise these values should 
be increased while a lower noise level would allow for these thresholds to be decreased.

Since the GN-C algorithm has been designed to terminate at the maximally resolved image by adjusting the number of CGLS iterations in the latter part of the GN-C algorithm, it is important to terminate the algorithm based on the norm $\eta_{n}$. If a fixed number of iterations is used the optimal number of iterations might be exceeded, and a point reached where the algorithm attempts to fit the solution to the unwanted noise-component in the measured data. When the GN-C algorithm reaches the point where it starts to fit the solution to the noise component of the measured data this will usually result in the value of $\eta_{n}$ starts to oscillate around some fixed value. This oscillation, in turn, will cause the value of $\Delta \eta_{n}$ to become positive (larger than -3\%) and the algorithm should therefore be terminated.

\section{Safeguard}

To avoid the GN-C algorithm from getting trapped in a local minimum or an oscillating mode, a safeguard based on the twonorm of the update vector has been implemented. The two-norm of the update values are not allowed to exceed one quarter of the two-norm of the vector holding the $k^{2}$ values. To prevent this, the update vector $\Delta k_{n}^{2}$ is multiplied by a scaling factor $\alpha_{\mathrm{CGLS}}$ determined by

$$
\alpha_{\mathrm{CGLS}}= \begin{cases}1 & \text { for } \frac{\left\|\underline{k}_{n}^{2}\right\|_{2}}{\left\|\underline{\Delta k_{n}^{2}}\right\|_{2}}>4 \\ \frac{\left\|\underline{k}_{n}^{2}\right\|_{2}}{4 \| \underline{\Delta k_{n}^{2} \|_{2}}} & \text { for } \frac{\left\|\underline{k}_{n}^{2}\right\|_{2}}{\| \underline{\Delta \underline{k}_{n}^{2} \|_{2}}}<4\end{cases}
$$

and the value of the vector $\underline{k}^{2}$ is updated using

$$
\underline{k}_{n+1}^{2}=\underline{k}_{n}^{2}+\alpha_{\mathrm{CGLS} \Delta \underline{k}_{n}^{2}} .
$$

The choice of a factor of 4 between the two-norm of the update vector and the two-norm of the vector holding the $k^{2}$ values has been chosen from an observation of when the algorithm fails and a considerable margin has been added. In our experience, for most cases the two-norm of the update vector is well below the limit and in the vast majority of cases where it exceeds the limit the algorithm still performs well without the scaling. In some instances, however, the update values can behave quite erratically and the scaling is necessary. When the scaling is applied, there has been no example of the algorithm failing, even though a large number of images of both simulations, phantom measurements, and patient measurements have been reconstructed. If anything, the scaling might be too restrictive, and a more advanced analysis of the update procedure may provide the means for determining it in a more sophisticated manner, thus allowing even faster convergence of the algorithm.

\section{Summary of Gauss-Newton CGLS Algorithm}

Combining these steps, the GN-C algorithm has the following steps in each iteration.

1) The forward model is used to calculate the electric fields from the distribution of constitutive parameters $\underline{k}_{n}^{2}$ and to calculate the value of $\eta_{n}$. The value of $\eta_{n}$ is used to check for termination and to check for the number of iterations to be used in the CGLS algorithm;

2) Calculation of the Jacobian $\underline{\underline{J}}$ for the distribution $\underline{k}_{n}^{2}$;

3) Use the CGLS algorithm to calculate the update value $\left[\underline{\Delta}_{n}^{2}\right]^{(m)}$ with $m=2$ or $m=16$ depending on the value of $\Delta \eta_{n}$ and update the values of the constitutive parameters using

$$
\underline{\Delta k_{n+1}^{2}}=\underline{k}_{n}^{2}+\alpha_{\mathrm{CGLS}}\left[\underline{\Delta k_{n}^{2}}\right]^{(m)} .
$$

As with the GN-T algorithm, the most time consuming part of the iterations is the calculation of the forward solution, taking approximately $1 \mathrm{~min}$ while the update step takes less than $1 \mathrm{~s}$. No measurable difference has been found in the overall time consumption per Newton iteration for the GN-T and the GN-C algorithms.

\section{Test OF Algorithm}

In this section, the performance of the GN-C algorithm is compared to the performance of the previously used GN-T algorithm using simulation, phantom measurement, and patient data.

\section{A. Simulated Data}

A simple 2-D target has been simulated and the images reconstructed using the GN-C algorithm compared with those obtained using the GN-T algorithm. The target was a circular scatterer with a radius of $2 \mathrm{~cm}$ and constitutive parameters $\epsilon_{r, \mathrm{t}}=50$ and $\sigma_{\mathrm{t}}=1.6 \mathrm{~S} / \mathrm{m}$ in a background medium with constitutive parameters $\epsilon_{r, \mathrm{bg}}=30$ and $\sigma_{\mathrm{bg}}=1.163 \mathrm{~S} / \mathrm{m}$. The target was centered at $(x, y)=(0,2 \mathrm{~cm})$ and a schematic of the setup is shown in Fig. 4. The 16 point-source antennas were positioned equidistantly about a $15.2 \mathrm{~cm}$ diameter circle concentrically surrounding the $14.0 \mathrm{~cm}$ diameter imaging zone and the frequency was $1000 \mathrm{MHz}$. The simulated measurement data had Gaussian noise added with an amplitude mimicking a noise floor of -100 $\mathrm{dBm}$.

The images obtained using the two different algorithms are shown in Fig. 5. The images are seen to be close to identical with the GN-C algorithm reaching a slightly higher maximum value than the GN-T algorithm. Both techniques recover the target quite well in both permittivity and conductivity. The background variation in both conductivity images is quite similar and a direct consequence of the simulated noise which is much higher than that encountered in practice. The value of the normalized two-norm $\eta_{n}$ of the two algorithms is shown in Fig. 6 along with $\Delta \eta_{n}$ for the GN-C algorithms. The GN-C algorithm was terminated after 11 iterations because the value of $\eta_{n}$ decreases to a value less than 0.03 . The sharp decrease in $\Delta \eta_{n}$ for the GN-C algorithm at iteration 11 indicates that the algorithm has transitioned from performing two to sixteen iterations in the CGLS algorithm. The value of $\eta_{n}$ decreases more quickly in the first few iterations, and subsequently levels out. The value of $\eta_{n}$ for the GN-T algorithm reaches a stable level after 13 iterations, and the image does not change significantly during the last seven iterations. The final errors for each are negligibly different which is not surprising given the similarity of the final images. 


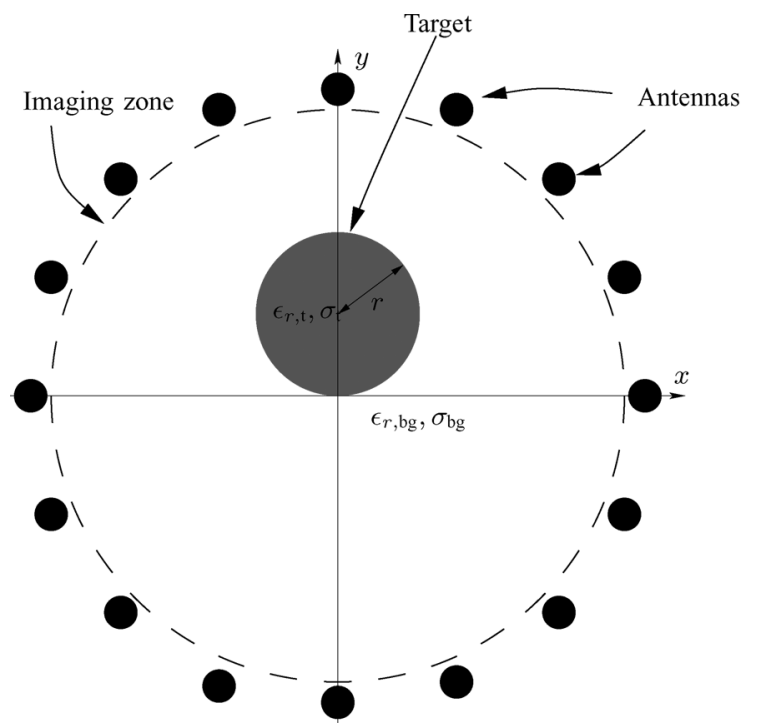

Fig. 4. Schematic of the setup of the simulation. The background has the constitutive parameters $\epsilon_{r, \mathrm{bg}}=30$ and $\sigma_{\mathrm{bg}}=1.163 \mathrm{~S} / \mathrm{m}$. The circular target is centered at $(x, y)=(0,2 \mathrm{~cm})$ and has the radius $r=2 \mathrm{~cm}$. The constitutive parameters of the target are $\epsilon_{r, \mathrm{t}}=50$ and $\sigma_{\mathrm{t}}=1.6 \mathrm{~S} / \mathrm{m}$.

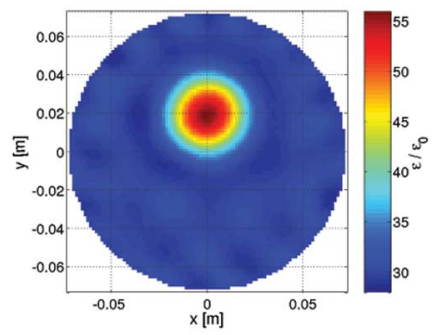

(a) GN-C, perm.

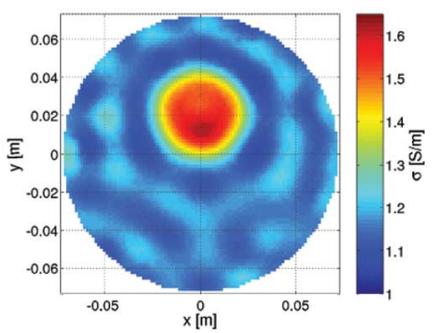

(c) GN-C, cond.

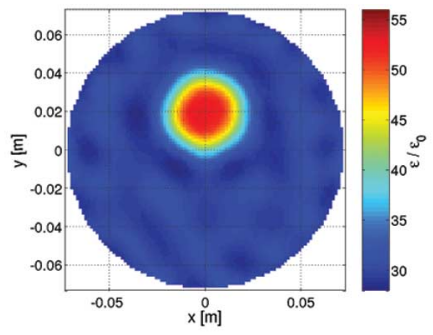

(b) GN-T, perm.

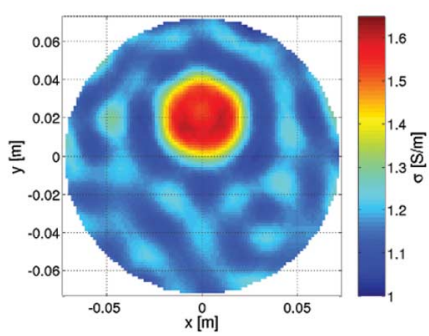

(d) GN-T, cond.
Fig. 5. Comparison of the reconstructed values of the conductivity and permittivity of the simulation case for the GN-C and the GN-T algorithms. The GN-C algorithm reached convergence after 11 iterations while the result of the GN-T algorithm is that of the 20th iteration. (a) GN-C, perm. (b) GN-T, perm. (c) GN-C, cond. (d) GN-T, cond.

To quantitatively compare the reconstructed values with the actual values, transects of the images for the two pairs along the $y$ axis are shown in Fig. 7. The results obtained with the two different algorithms are quite similar. The permittivity reconstructed using the GN-T algorithm seems to be a slightly better fit to the true value with less overshoot of the central target value while no clear difference is seen in the images of the conductivity.

In Fig. 8, the L-curve for the CGLS algorithm for the linear update problem at the first iteration of the GN-C algorithm is plotted against the L-curve that would be obtained using

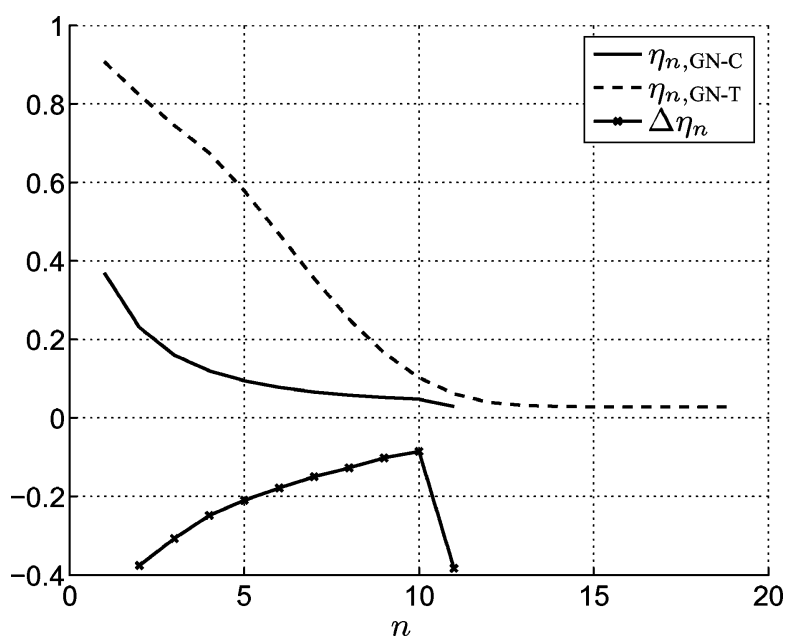

Fig. 6. Normalized two-norm $\eta_{n}$ for the two algorithms and $\Delta \eta_{n}$ for the GN-C algorithm.

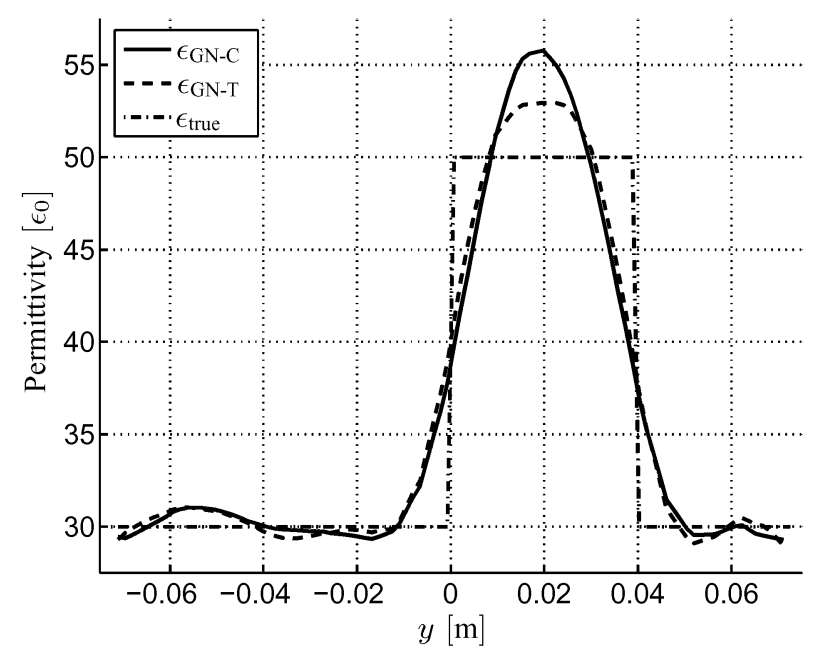

(a)

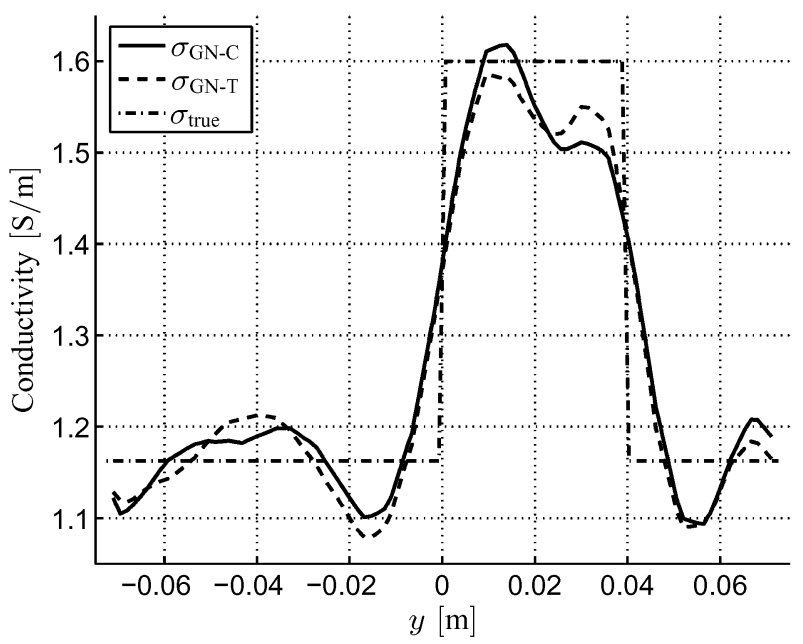

(b)

Fig. 7. Comparison of the reconstructed values along the $y$ axis. (a) Permittivity; (b) conductivity.

Tikhonov regularization for the same problem. The solutions obtained using the CGLS algorithm are seen to follow those 


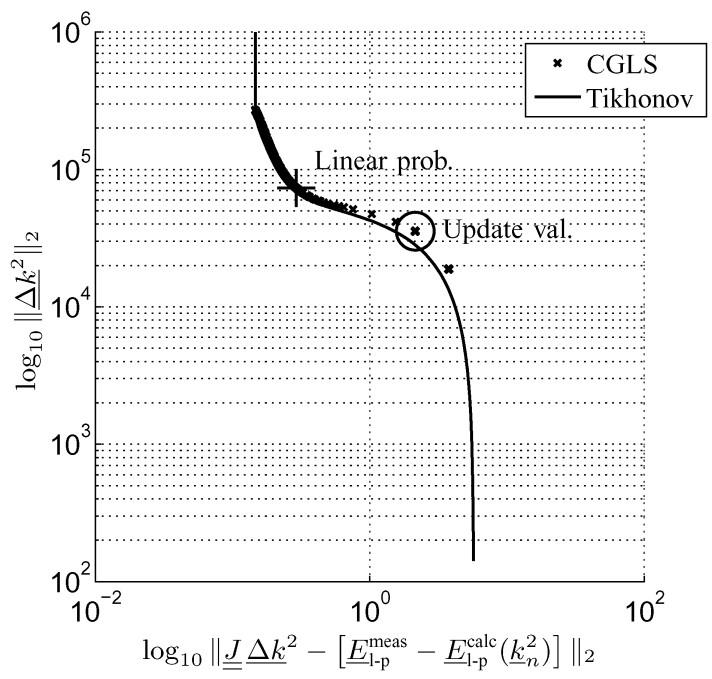

Fig. 8. Comparison of the L-curve obtained using the CGLS algorithm and the L-curve obtained using the Tikhonov regularization algorithm. The solution considered to be optimal for a linear problem is indicated by the + and is obtained after 25 iteration of the CGLS algorithm. The solution used to update the values in the GN-C algorithm is that obtained after two iterations of the CGLS algorithm and is indicated by the circle.

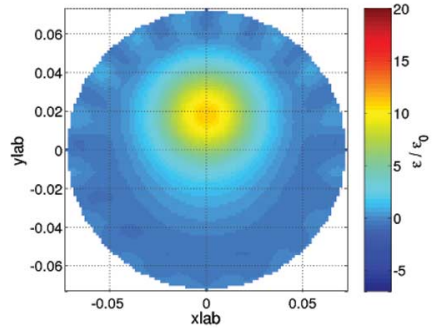

(a) Perm., 2 iterations

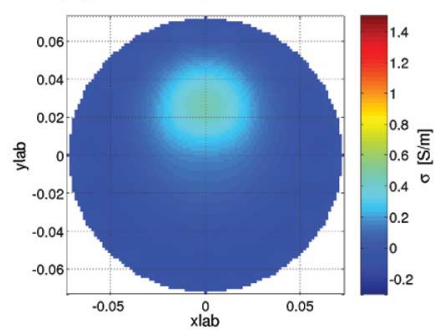

(c) Cond., 2 iterations

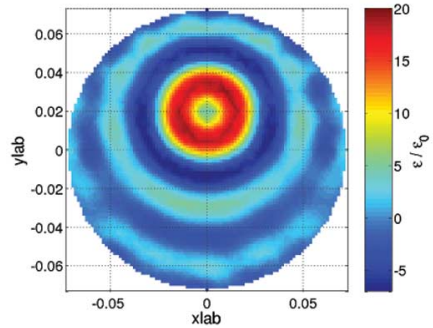

(b) Perm., 25 iterations

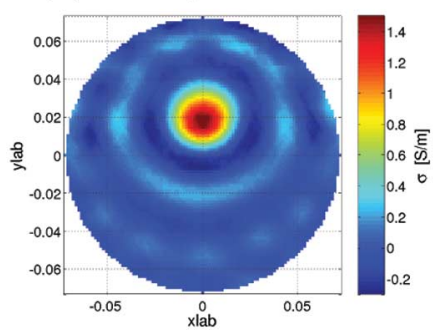

(d) Cond., 25 iterations
Fig. 9. Comparison of the update vector $\left[\Delta k_{1}^{2}\right]^{(2)}$ found using 2 iterations of the CGLS algorithm and the update vector $\left[\underline{\Delta k}_{1}^{2}\right]^{(25)}$ found using 25 iterations. (a) Perm., 2 iterations; (b) perm., 25 iterations; (c) cond., 2 iterations; (d) cond., 25 iterations.

obtained with the Tikhonov algorithm closely, with the first iteration yielding the point in the lower right part of the curve. The solution after two iterations, which is used to update the values of the constitutive parameters in the GN-C algorithm is indicated by the circle in the plot. This is quite far from the solution closest to the point where the L-curve has its maximum curvature, the solution which is considered to be optimal when dealing with linear problems [10, Sec. 7.5]. This point is achieved after 25 CGLS iterations and is marked with a "+" in Fig. 8. The update vectors $\left[\underline{\Delta} k_{1}^{2}\right]^{(2)}$ and $\left[\underline{\Delta k_{1}^{2}}\right]^{(25)}$ obtained after 2 and 25 CGLS iterations, respectively, are shown in Fig. 9. In this figure, the elements of the vectors have been assigned to the corresponding coordinate positions, yielding

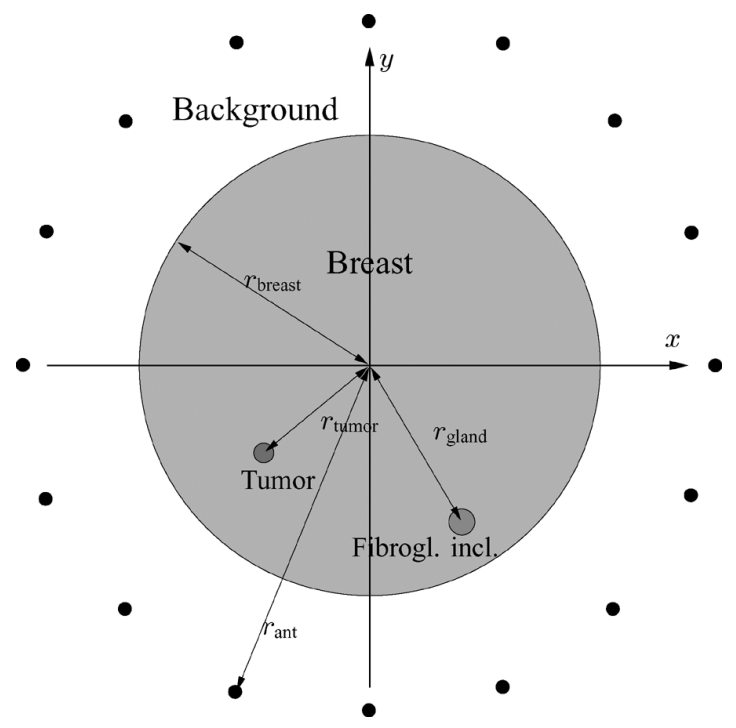

Fig. 10. Breast phantom configuration. The cylindrical breast phantom had a relative permittivity of $\epsilon_{r, \text { breast }}=12.6$, a conductivity of $\sigma_{\text {breast }}=0.62 \mathrm{~S} / \mathrm{m}$, and a radius of $r_{\text {breast }}=5 \mathrm{~cm}$. The $28 \mathrm{~mm}$ tumor inclusion had its center $r_{\text {tumor }}=3 \mathrm{~cm}$ from the center of the breast phantom with $\epsilon_{r, \text { tumor }}=53.4$ and $\sigma_{\text {tumor }}=1.15 \mathrm{~S} / \mathrm{m}$. The $21 \mathrm{~mm}$ fibroglandular-tissue inclusion was positioned with its center $r_{\text {gland }}=4 \mathrm{~cm}$ and $\epsilon_{r, \text { sland }}=32.7$ and $\sigma_{\text {gland }}=1.28 \mathrm{~S} / \mathrm{m}$. The antennas were positioned in a circular array with a radius of $r_{\text {ant }}=7.5 \mathrm{~cm}$ and the coupling liquid filling out the background had constitutive parameters $\epsilon_{r, \mathrm{bg}}=23.3$ and $\sigma_{\mathrm{bg}}=1.13 \mathrm{~S} / \mathrm{m}$. The imaging zone was a $13.5 \mathrm{~cm}$ diameter circle.

images showing the spatial distribution of the updates. It is seen that the updates found using two iterations recover the shape of the circular target nicely and has virtually no spatial oscillations in the updates. The updates found using 25 iterations, corresponding to the point on the L-curve with maximum curvature, still detects the target but with many more spatial oscillations present in the update values. The center of the recovered object also has a reduced property artifact. The $m=25$ solution is therefore not suitable for updating the $\underline{k}^{2}$ vector.

\section{B. Fatty Breast Phantom}

To illustrate the details in the new algorithm and the impact on the image quality when the algorithms are applied to reconstruct tomographic images from measured data, phantom data was acquired at $1100 \mathrm{MHz}$. A schematic representation of the phantom is shown in Fig. 10. The phantom consisted of a $10 \mathrm{~cm}$ diameter thin-walled plastic cylinder filled with a glycerin-water mixture with constitutive parameters $\epsilon_{r \text {,breast }}=12.6$ and $\sigma_{\text {breast }}=0.62 \mathrm{~S} / \mathrm{m}$ simulating a primarily fatty breast. Inside the breast phantom, two smaller cylinders were positioned with liquids simulating fibroglandular tissue and a tumor. The $28 \mathrm{~mm}$ diameter tumor inclusion had constitutive parameters $\epsilon_{r, \text { tumor }}=53.4$ and $\sigma_{\text {tumor }}=1.15 \mathrm{~S} / \mathrm{m}$ while the $21 \mathrm{~mm}$ diameter fibroglandular inclusion was approximated by a liquid with $\epsilon_{r, \text { gland }}=32.7$ and $\sigma_{\text {gland }}=1.28 \mathrm{~S} / \mathrm{m}$. The center of the tumor was positioned approximately $3 \mathrm{~cm}$ from the center of the breast with its center at $\left(x_{\text {tumor }}, y_{\text {tumor }}\right) \approx(-2.3 \mathrm{~cm},-1.9 \mathrm{~cm})$ while the fibroglandular inclusion had its center at $\left(x_{\text {gland }}, y_{\text {gland }}\right) \approx$ $(2 \mathrm{~cm},-3.5 \mathrm{~cm})$, which is approximately $4 \mathrm{~cm}$ from the breast center. The coupling liquid had a relative permittivity of $\epsilon_{r, \mathrm{bg}}=$ 23.4 and a conductivity of $\sigma_{\mathrm{bg}}=1.13 \mathrm{~S} / \mathrm{m}$. 


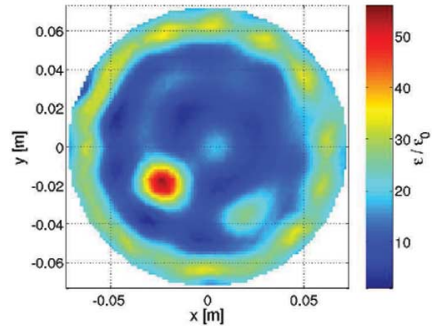

(a) GN-C, perm.

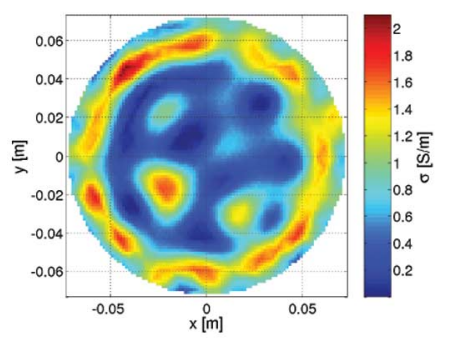

(c) GN-C, cond.

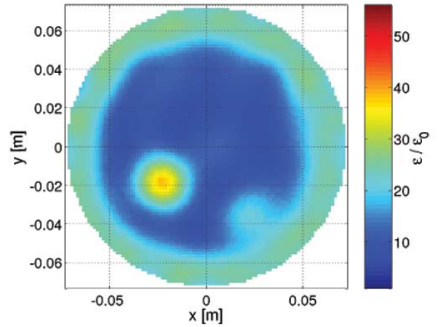

(b) GN-T, perm.

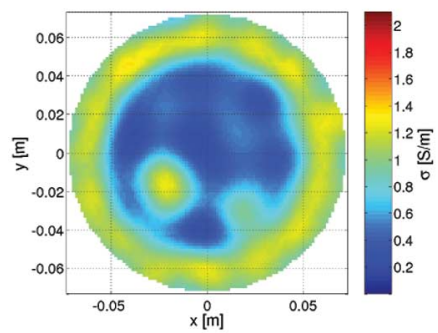

(d) GN-T, cond.
Fig. 11. Comparison of the reconstructed values of the conductivity and permittivity for the GN-C and the GN-T algorithms. The GN-C algorithm reached convergence after 11 iterations while the result of the GN-T is that reached after 20 iterations. (a) GN-C, perm. (b) GN-T, perm. (c) GN-C, cond. (d) GN-T, cond.

The results of the inversion with the GN-C and the GN-T algorithms are shown in Fig. 11. The perimeter of the breast phantom is readily visible in both cases, with a higher degree of artifacts outside the phantom for the GN-C images. Both the size and contrast of the two inclusions are reconstructed better with the GN-C algorithm than with the GN-T algorithm. In contrast, the amplitude of the artifacts within the recovered breast phantom are significantly elevated in the GN-C algorithm compared to that of the GN-T algorithm. The fibroglandular inclusion is localized well in the GN-C images while it appears to blur with the surrounding background in the associated GN-T images. This further illustrates that the increased spatial resolution of the GN-C algorithm comes at the expense of a higher level of the artifacts. By adjusting the number of iterations of the CGLS algorithm in the latter part of the GN-C algorithm, the balance between spatial resolution and artifacts can be adjusted with fewer iterations yielding lower spatial resolution and more iterations yielding higher level of the artifacts.

The normalized two-norm $\eta_{n}$ for the GN-C and GN-T algorithms as function of the iteration number are shown in Fig. 12. As was observed for the simulation case, it is readily seen that the value of $\eta_{n}$ declines much faster for the GN-C than for the GN-T algorithm.

\section{Patient Measurements}

Imaging the breast with the 2-D imaging system poses inherent challenges. For the planes closest to the chest wall, the possibility of artifacts arises due to the proximity of higher water content tissue associated with the pectoral muscles and the rib cage. For the planes closest to the nipple, the breast is more conical than cylindrical, posing different challenges for this system. It is therefore of interest to examine the performance of the new algorithm close to the chest wall, at the middle of the breast, and close to the anterior part of the breast.

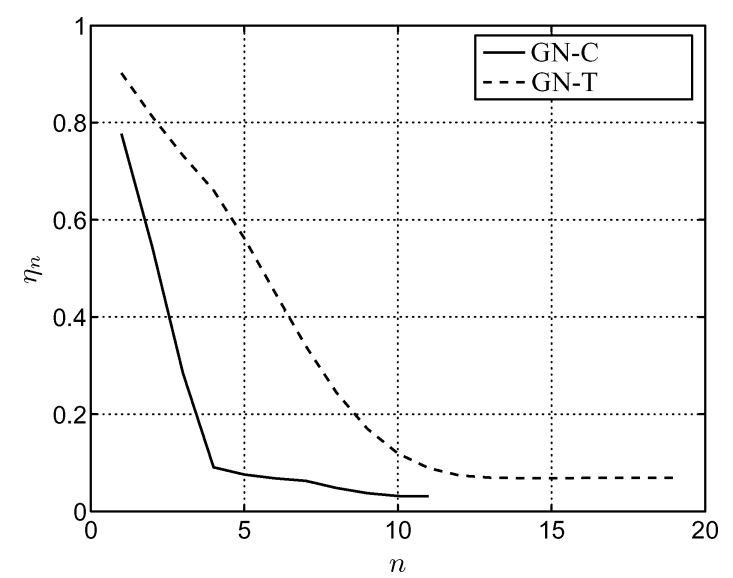

Fig. 12. Comparison of the normalized two-norm $\eta_{n}$ for the GN-C and GN-T algorithms.

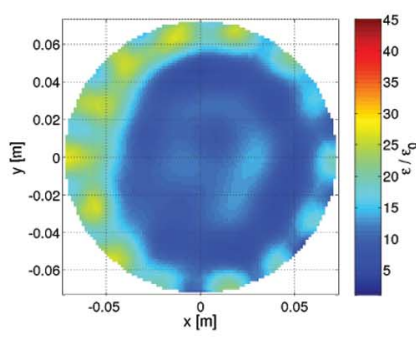

(a) Plane 1, perm

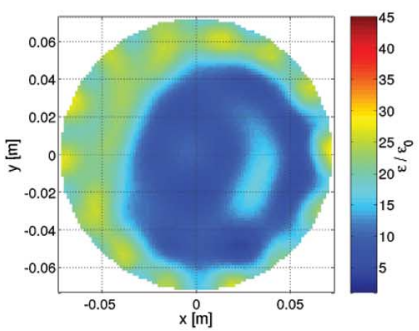

(c) Plane 4, perm.

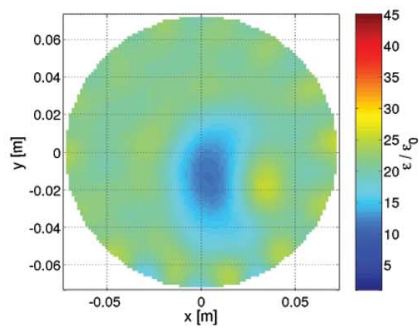

(e) Plane 7, perm.

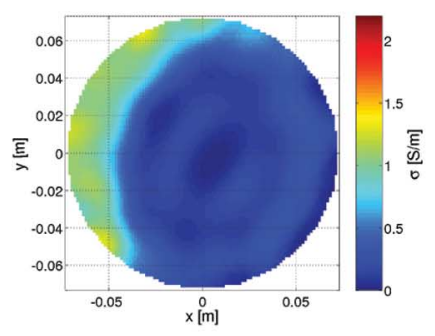

(b) Plane 1, cond.

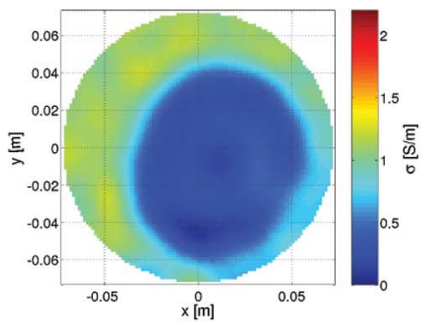

(d) Plane 4, cond.

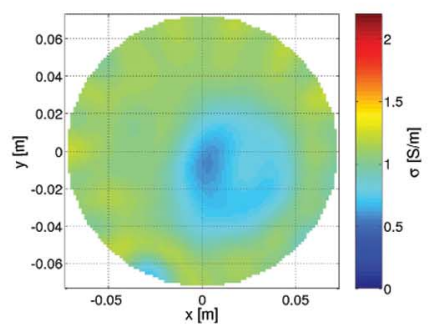

(f) Plane 7, cond.
Fig. 13. Results obtained using the GN-T algorithm for the left breast of the test patient at three of the seven planes. All images are created using 20 iterations of the GN-T algorithm. (a) Plane 1, perm. (b) plane 1, cond. (c) plane 4, perm. (d) plane 4, cond. (e) plane 7, perm. (f) plane 7, cond.

Fig. 13 shows the results obtained at $1100 \mathrm{MHz}$ with the GN-T algorithm for planes 1, 4, and 7 (with plane 1 being closest to the chest wall) for the left breast, while the images for the right breast are shown in Fig. 15.

The corresponding images obtained using the GN-C algorithm are shown in Figs. 14 and 16, respectively. The results 


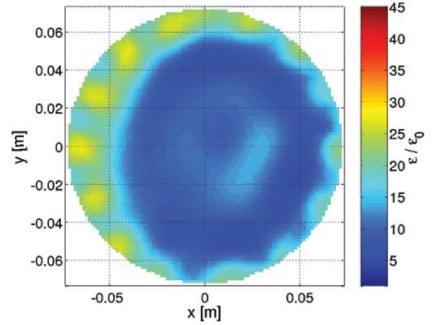

(a) Plane 1, perm

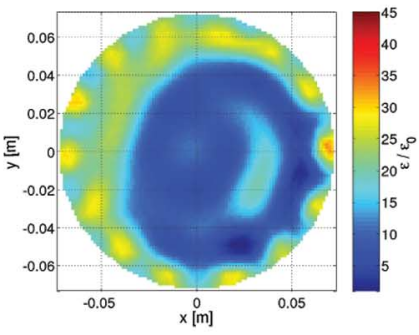

(c) Plane 4, perm.

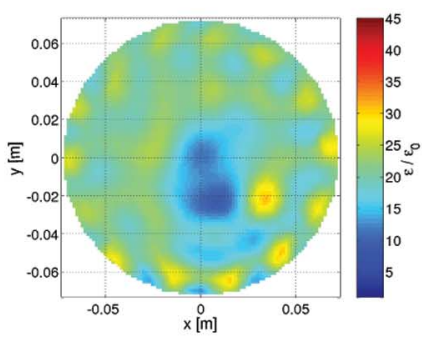

(e) Plane 7, perm.

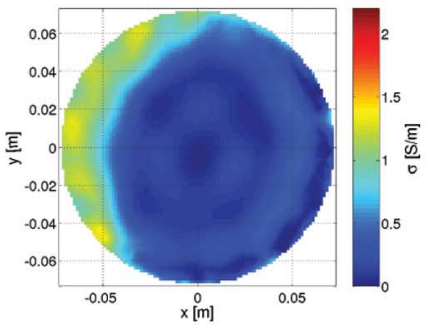

(b) Plane 1, cond.

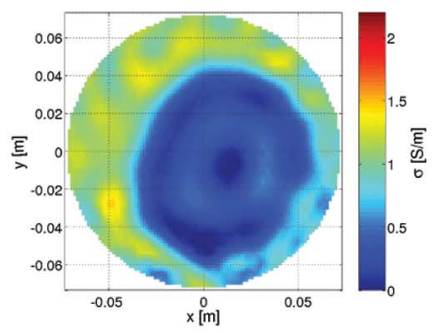

(d) Plane 4, cond.

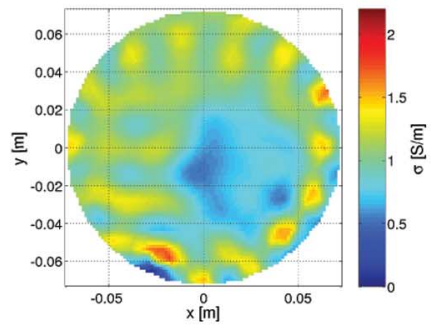

(f) Plane 7 , cond.

Fig. 14. Results obtained using the GN-C algorithm for the left breast of the test patient at three of the seven planes. The algorithm converge and terminates after 10 iterations of the algorithm for all three planes. (a) Plane 1, perm. (b) plane 1 , cond. (c) plane 4 , perm. (d) plane 4 , cond. (e) plane 7 , perm. (f) plane 7 , cond.

shown for the GN-T algorithm were reconstructed in 20 iterations while the GN-C algorithm reached convergence after 10 iterations for all three planes of the left breast and after 11 iterations for plane 1, 9 iterations for plane 4 , and 11 iterations for plane 7 of the right breast.

The patient in this case was 36 years old, had scattered-density breasts and was imaged with an 80:20 glycerin:water coupling fluid. The tumor was distributed over roughly a $4 \mathrm{~cm}$ diameter zone located near the anterior of the right breast at a 7 clock-face orientation, viewing the patient en face. The images for the two methods are quite similar providing a level of confidence for the overall approach. In general, the breast properties are lower than those of the background medium, with the complete perimeter of the breast in plane 1 not entirely visible-most likely due to the breast cross section being either larger than the imaging zone or positioned too close to the antenna array. The conductivity images for plane 1 are quite homogeneous within the breast perimeter. The permittivity images show scattered zones of slightly elevated properties, corresponding to scattered fibroglandular zones. The differences between the GN-C and GN-T algorithm images are minimal for this plane.

The full outline of the breast is more obviously discerned at the fourth imaging plane. Both the permittivity and conductivity

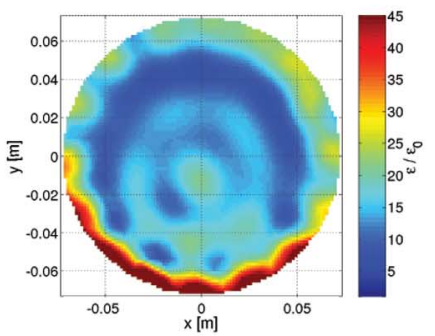

(a) Plane 1, perm

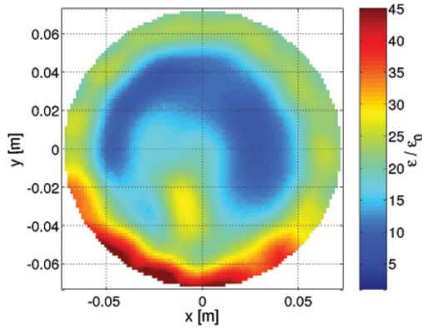

(c) Plane 4, perm.

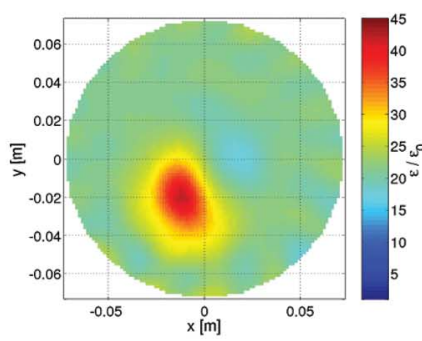

(e) Plane 7, perm.

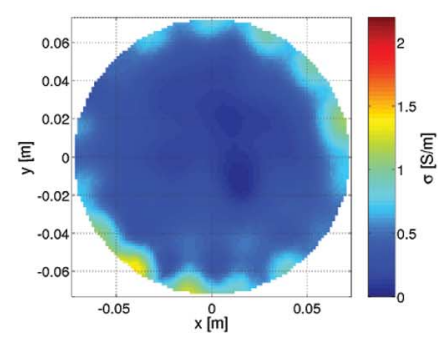

(b) Plane 1, cond.

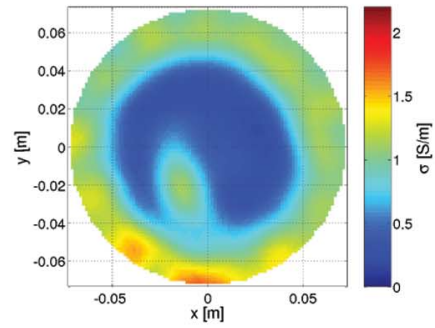

(d) Plane 4, cond.

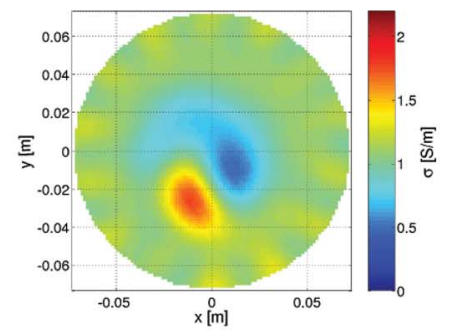

(f) Plane 7, cond.
Fig. 15. Results obtained using the GN-T algorithm for the right breast of the test patient at three of the seven planes. All images are created using 20 iterations of the algorithm. (a) Plane 1, perm. (b) plane 1, cond. (c) plane 4, perm. (d) plane 4, cond. (e) plane 7, perm. (f) plane 7, cond.

images display elevated zones throughout the breast cross section associated with fibroglandular tissue. The elevated zones for the right breast appear to be more concentrated in the lower left quadrant, suggesting some influence from the tumor. This feature seems to be accentuated more by the GN-C algorithm. Likewise, a crescent-shaped feature to the right of the permittivity image of the left breast is also more accentuated in the GN-C images. While this is most likely due to the presence of fibroglandular tissue, it may be an example of the GN-C algorithm overshooting the recovered property values similar to the simulation case above. Of less importance but still noticeable is that the recovered background distribution (i.e., outside the breast perimeter) is more uneven for the GN-C algorithm.

The images for plane 7 also provide useful information about the patient. Obviously the overall breast cross section is smaller than for the previous planes. In addition, similar to that of plane 4 , the recovered background distribution is fairly uneven - more so in the GN-C images than in the GN-T counterpart. For the most part, both left breast conductivity images show minimal variation within the breast. The corresponding permittivity images show an elevated zone to the right which may be associated with the higher properties of the nipple and concentrations of fibroglandular tissue. The elevated zone in the GN-C image is more localized with a higher central value than that 


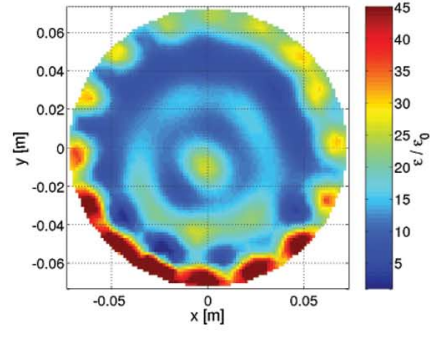

(a) Plane 1, perm

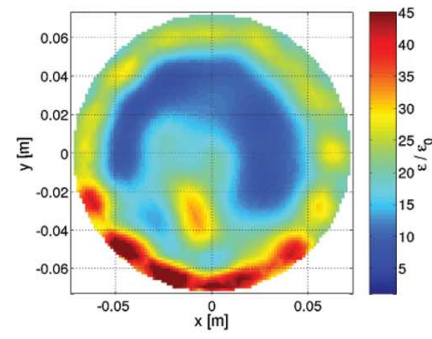

(c) Plane 4, perm.

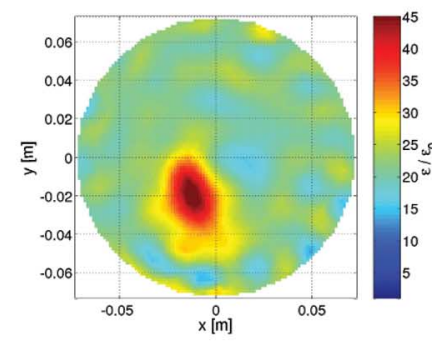

(e) Plane 7, perm.

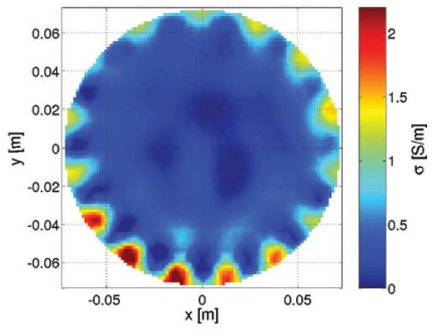

(b) Plane 1, cond.

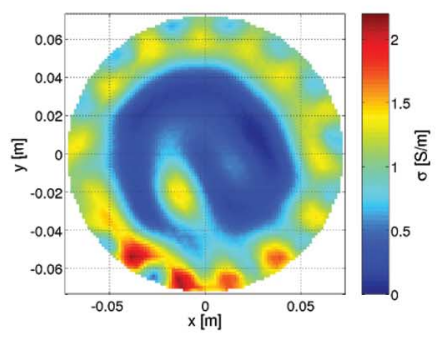

(d) Plane 4, cond.

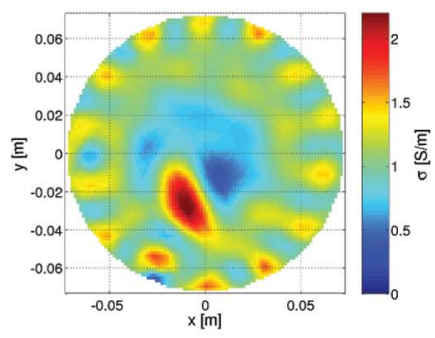

(f) Plane 7, cond.
Fig. 16. Results obtained using the GN-C algorithm for the right breast of the test patient at three of the seven planes. The algorithm converges and terminates after 11 iterations for plane 1 and plane 7, and after 9 iterations for plane 4. (a) Plane 1, perm. (b) plane 1, cond. (c) plane 4, perm. (d) plane 4, cond. (e) plane 7, perm. (f) plane 7 , cond.

for the GN-T image which appears partially blurred into the surrounding background distribution which is slightly elevated compared with the overall breast properties. The plane 7 images of the right breast all show localized property-enhanced zones in the lower left quadrant associated with the tumor location. The overall breast outline is vaguely visible within the background because of the limited breast/coupling liquid contrast there. The reconstructed tumor zones are more readily distinguished in the GN-C images because the recovered properties are higher.

In addition to the patient data presented in this paper, a number of other reconstructions of patient data have been carried out, and in general the GN-C algorithm is better able to extract internal features at the cost of overall enhanced image artifacts. The increased artifact level is a result of the GN-C algorithm, in the process of extracting all available information from the measurement data, includes an increased amount of the signal noise in the reconstructed image. The enhanced artifacts affects the reconstructed images in two ways. First, the calculated signal changes because of the artifacts. If the reconstruction algorithm is not terminated, this may lead to the algorithm attempting to fit the reconstructed image to a solution in which the noise, and thereby the artifacts, are dominant, yielding unpredictable results and useless images. Second, the enhanced artifacts inside the breast may be interpreted as tumors, thereby causing a false cancer-detection. It is therefore of great importance to terminate the GN-C algorithm as described in Section IV-B.

As mentioned earlier, a significant advantage of the GN-C algorithm is the number of iterations required to reconstruct the images. Given the fact that the time needed to complete one iteration is the same for both algorithms, the use of the GN-C algorithm reduced the overall time consumption by $45 \%$ to $55 \%$.

\section{CONCLUSION}

A new algorithm for determining the update values of the constitutive parameters in an iterative Gauss-Newton algorithm for microwave imaging of the breast has been derived. The algorithm is based on the use of the CGLS algorithm for solving the linear problem arising when solving for the image update values. The algorithm has been implemented as a two-stage procedure in which the first iterations of the Gauss-Newton algorithm are used to extract a coarse estimate of the distribution of constitutive parameters in the imaging domain while the latter set are used for extraction of finer details.

When compared to the previously used inversion algorithm, it appears to detect small objects more reliably at the cost of increased image artifacts. The artifacts can potentially be problematic in breast screening by increasing the number of false cancer detections. Further research is currently being pursued with the aim to reduce the level of the artifacts without sacrificing the increased spatial resolution.

Finally, the GN-C has been shown to use fewer iterations to converge, thus reducing the time-consumption by as much as $55 \%$.

\section{REFERENCES}

[1] X. Li, E. Bond, B. Van Veen, and S. Hagness, "An overview of ultrawideband microwave imaging via space-time beamforming for earlystage breast-cancer detection," IEEE Antennas Propag. Mag. , vol. 47, no. 1, pp. 19-34, 2005.

[2] E. C. Fear, P. Meaney, and M. Stuchly, "Microwaves for breast cancer detection?," IEEE Potentials, vol. 22, no. 1, pp. 12-18, 2003.

[3] T. Williams, E. C. Fear, and D. Westwick, "Tissue sensing adaptive radar for breast cancer detection: Investigations of reflections from the skin," in IEEE Antennas and Propagation Society Symp. in conjunction with USNC/URSI National Radio Science Meeting and IEEE Antennas and Propagation Soc. AP-S Int. Symp. Digest, 2004, vol. 3, pp. 2436-2439.

[4] J. Sill and E. C. Fear, "Tissue sensing adaptive radar for breast cancer detection: Preliminary experimental results," in IEEE Int. Microwave Symp. MTT-S Digest, 2005, p. 4.

[5] J. Sill and E. C. Fear, "Tissue sensing adaptive radar for breast cancer detection: Study of immersion liquids," Electron. Lett., vol. 41, no. 3, pp. 113-115, 2005.

[6] J. M. Sill and E. C. Fear, "Tissue sensing adaptive radar for breast cancer detection-experimental investigation of simple tumor models," IEEE Trans. Microw. Theory Tech., vol. 53, no. 11, pp. 3312-3319, 2005.

[7] R. Nilavalan, J. Leendertz, I. Craddock, A. Preece, and R. Benjamin, "Numerical analysis of microwave detection of breast tumours using synthetic focussing techniques," in Proc. IEEE Antennas and Propagation Society Symp., 2004, vol. 3, pp. 2440-2443.

[8] A. Bulyshev, A. Souvorov, S. Semenov, R. Svenson, A. Nazarov, Y. Sizov, and G. Tatsis, "Three-dimensional microwave tomography. Theory and computer experiments in scalar approximation," Inverse Problems, vol. 16, no. 3, pp. 863-875, 2000. 
[9] S. Semenov, R. Svenson, A. Bulyshev, A. Souvorov, A. Nazarov, Y. Sizov, V. Posukh, A. Pavlovsky, P. Repin, A. Starostin, B. Voinov, M. Taran, G. Tatsis, and V. Baranov, "Three-dimensional microwave tomography: Initial experimental imaging of animals," IEEE Trans. Biomed. Eng., vol. 49, no. 1, pp. 55-63, 2002.

[10] P. C. Hansen, Rank-Deficient and Discrete Ill-Posed Problems: Numerical Aspects of Linear Inversion, ser. Monographs on Mathematical Modeling and Computation. Philadelphia, PA: SIAM, 1998.

[11] Q. Fang, P. Meaney, S. Geimer, A. Streltsov, and K. Paulsen, "Microwave image reconstruction from 3-D fields coupled to 2-D parameter estimation," IEEE Trans. Med. Imaging, vol. 23, no. 4, pp. 475-484, 2004.

[12] Q. Fang, "Computational methods for microwave medical imaging," Ph.D. dissertation, Thayer School of Engineering, Dartmouth College, Hanover, NH, 2004.

[13] P. Meaney, S. Pendergrass, M. Fanning, D. Li, and K. Paulsen, "Importance of using a reduced contrast coupling medium in $2 \mathrm{D}$ microwave breast imaging," J. Electromagn. Waves Applicat., vol. 17, no. 2, pp. 333-355, 2003.

[14] D. Li, P. M. Meaney, T. Raynolds, S. A. Pendergrass, M. W. Fanning, and K. D. Paulsen, "Parallel-detection microwave spectroscopy system for breast imaging," Rev. Scientific Instruments, vol. 75, no. 7, pp. 2305-2313, 2004.

[15] P. Meaney, K. Paulsen, S. Geimer, S. Haider, and M. Fanning, "Quantification of 3-D field effects during 2-D microwave imaging," IEEE Trans. Biomed. Eng., vol. 49, no. 7, pp. 708-720, 2002.

[16] P. Meaney, K. Paulsen, B. Pogue, and M. Miga, "Microwave image reconstruction utilizing log-magnitude and unwrapped phase to improve high-contrast object recovery," IEEE Trans. Med. Imaging, vol. 20, no. 2, pp. 104-116, 2001.

[17] C. T. Kelley, Iterative Methods for Linear and Nonlinear Equations, ser. Frontiers in Applied Mathematics. Philadelphia, PA: SIAM, 1995, vol. 16.

[18] C. T. Kelley, Solving Nonlinear Equations With Newton's Method. Philadelphia, PA: SIAM, 2003.

[19] P. Meaney, K. Paulsen, A. Hartov, and R. Crane, "Microwave imaging for tissue assessment: Initial evaluation in multitarget tissue-equivalent phantoms," IEEE Trans. Biomed. Eng., vol. 43, no. 9, pp. 878-890, 1996.

[20] K. Paulsen, P. Meaney, M. Moskowitz, J. Sullivan, and J. M. , "A dual mesh scheme for finite element based reconstruction algorithms," IEEE Trans. Biomed. Eng., vol. 14, no. 3, pp. 504-514, 1995.

[21] K. Paulsen and P. Meaney, "Nonactive antenna compensation for fixedarray microwave imaging-Part I: Model development," IEEE Trans. Biomed. Eng., vol. 18, no. 6, pp. 496-507, 1999.

[22] P. Meaney, K. Paulsen, J. Chang, M. Fanning, and A. Hartov, "Nonactive antenna compensation for fixed array microwave imaging-Part II: Imaging results," IEEE Trans. Biomed. Eng., vol. 18, no. 6, pp. 508-518, 1999.

[23] N. Joachimowicz, C. Pichot, and J. Hugonin, "Inverse scattering: An iterative numerical method for electromagnetic imaging," IEEE Trans. Antennas Propag., vol. 39, no. 121, pp. 1742-1753, 1991.

[24] Q. Fang, P. Meaney, and K. Paulsen, "Multi-dimensional phase unwrapping: Definition and properties," IEEE Trans. Image Processing, accepted for publication.

[25] P. Meaney, E. Demidenko, N. Yagnamurthy, D. Li, M. Fanning, and K. Paulsen, "A two-stage microwave image reconstruction procedure for improved internal feature extraction," Med. Phys., vol. 28, no. 11, pp. 2358-2369, 2001.

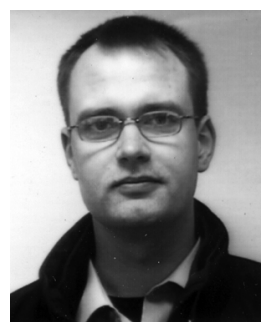

Tonny Rubæk (S'02) received the M.Sc.E.E. degree from the Technical University of Denmark, Lyngby, in 2004, where he is currently working toward the $\mathrm{Ph} . \mathrm{D}$. degree.

His research areas include linear and nonlinear microwave-imaging algorithms and associated hardware.

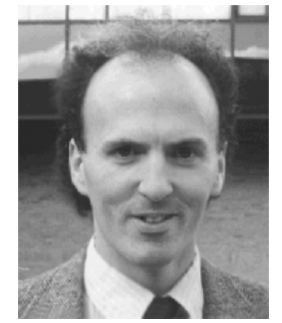

Paul M. Meaney (M'92) received the A.B. degree in both computer science and electrical engineering from Brown University, Providence, RI, in 1982, the M.S. degree in electrical engineering from the University of Massachusetts, Amherst, in 1985, and the $\mathrm{Ph} . \mathrm{D}$. degree in biomedical engineering from Dartmouth College, Hanover, NH, in 1995.

He was an NSF-NATO Postdoctoral Fellow at the Royal Marsden Hospital in Sutton, England, from 1996 to 1997. He was a Research Assistant Professor at Dartmouth College from 1997 to 2003 and a Research Associate Professor since 2003. He has three patents related to microwave imaging and is coauthor on over 120 journal articles and conference proceedings. His interests include developing microwave imaging for biomedical applications-especially breast imaging and hyperthermia monitoring.

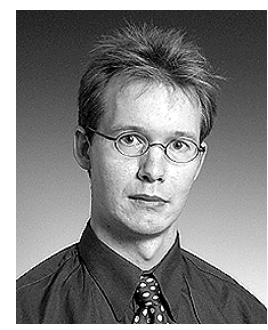

Peter Meincke (S'93-M'96) was born in Roskilde, Denmark, on November 25, 1969. He received the M.S.E.E. and Ph.D. degrees from the Technical University of Denmark (DTU), Lyngby, Denmark, in 1993 and 1996, respectively.

In spring and summer of 1995, he was a Visiting Research Scientist at the Electromagnetics Directorate of Rome Laboratory, Hanscom Air Force Base, MA. In 1997, he was with a Danish cellular phone company, working on theoretical aspects of radio-wave propagation. In spring and summer of 1998, he was visiting the Center for Electromagnetics Research at Northeastern University, Boston, MA, while holding a Postdoctoral position from DTU. In 1999, he became a staff member in the Department of Electromagnetic Systems, DTU. He is currently an Associate Professor with Ørsted-DTU, ElectroScience Section, DTU. His current teaching and research interests include electromagnetic theory and scattering, inverse problems, antenna theory, microwave imaging, and wireless communications.

Dr. Meincke won the First Prize Award in the 1996 IEEE Antennas and Propagation Society Student Paper Contest in Baltimore, MD, for his paper on uniform physical theory of diffraction equivalent edge currents. Also, he received the 2000 R. W. P. King Paper Award for his paper entitled, "Time-domain version of the physical theory of diffraction" published in the February 1999 issue of the IEEE TRANSACTIONS ON ANTENNAS AND PROPAGATION.

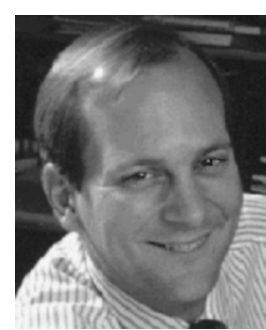

Keith D. Paulsen (S'85-M'86) received the B.S degree in biomedical engineering from Duke University, Durham, NC, in 1981 and the M.S. and $\mathrm{Ph} . \mathrm{D}$. degrees in biomedical engineering from Dartmouth College, Hanover, NH, in 1984 and 1986, respectively.

From 1986 to 1988, he was an Assistant Professor in the Electromagnetics Group within the Department of Electrical and Computer Engineering, University of Arizona, Tucson. He is currently a Professor at the Thayer School of Engineering, Dartmouth College and the Director of the Radiobiology and Bioengineering Research Program for the Norris Cotton Cancer Center within the Dartmouth-Hitchcock Medical Center, Lebanon, NH. His research interests include computational methods with particular emphasis on biomedical problems in cancer therapy and imaging, and model-guided surgery. 